\section{Response to: 'Associations of regular glucosamine use with all-cause and cause- specific mortality: causality assumptions need to be checked' by Safiri and Mansournia}

We appreciated Safiri and Mansournia for their interest and comment on our recent study. ${ }^{12}$ Safiri et al ${ }^{1}$ mentioned that we did not explain the steps for the selection of confounders that have been included in our fully adjusted models. We acknowledged that due to the limited number of the words for the manuscript required by the journal, the content for the explanation for selection of confounders was not included. Actually, all confounders in our study were selected according to the published literature, ${ }^{3-5}$ and these were the most important risk factors for mortality. In total, 27 confounders were included in our fully adjusted models, and the adjustment for confounding was sufficient.

As suggested by Safiri and Mansournia, ${ }^{1}$ we examined the balance of confounding variables between the glucosamine users and non-users by using standardised mean difference. The most of confounding variables were considered balanced between the two groups (table 1), suggesting that the application of propensity scores was appropriate. We evaluated the proportional hazard assumptions for propensity scores and the outcomes, and no evidence of a violation of the assumption was observed. When we put propensity scores into the models as a covariate to adjust for baseline differences, the results were not substantially changed.

The proportional hazard assumptions for glucosamine use and outcomes were evaluated for all models, and no violation of the assumption was found. Due to 10 models for five outcomes used in our study, we did not provide the hazard curves and $\mathrm{p}$ values in the article. ${ }^{2}$ However, we stated clearly that no violation of the assumption was found in the section of statistical analysis in the article. ${ }^{2}$

We did not provide $\mathrm{p}$ values for the differences in the baseline characteristics of the participants between the two groups due to a very large sample size. Even if there was a minor difference, the test also showed statistically significant results. All the $\mathrm{p}$ values for the differences in the baseline characteristics were less than 0.001 in this study, and all the baseline variables were included in the fully adjusted models.

Finally, in this study, we excluded participants who withdrew from the study (1299) and those with missing data on the use of glucosamine (6160). We do agree that differential reasons for loss to follow-up could lead to selection bias. However, the baseline characteristics of the participants excluded and included in this study were generally similar, and no differential reason for loss to follow-up was found.

\section{Zhi-Hao Li, Qing-Mei Huang, Chen Mao $\odot$}

Correspondence to Professor Chen Mao, Department of Epidemiology, School of Public Health, Southern Medical University, Guangzhou, China; maochen9@smu.edu.cn

Contributors All authors critically reviewed the manuscript for important intellectual content.

Funding The authors have not declared a specific grant for this research from any funding agency in the public, commercial or not-for-profit sectors.

Competing interests None declared.

Patient and public involvement Patients and/or the public were not involved in the design, conduct, reporting or dissemination plans of this research.

Patient consent for publication Not required.

Provenance and peer review Commissioned; internally peer reviewed.

(c) Author(s) (or their employer(s)) 2020. No commercial re-use. See rights and permissions. Published by BMJ.

\section{Check for updates}

To cite Li Z-H, Huang Q-M, Mao C. Ann Rheum Dis Epub ahead of print: [please include Day Month Year]. doi:10.1136/annrheumdis-2020-217896

Received 6 May 2020

Accepted 7 May 2020

\section{Linked}

- http://dx.doi.org/10.1136/annrheumdis-2020-217732

Ann Rheum Dis 2020;0:1-2. doi:10.1136/annrheumdis-2020-217896

ORCID iD

Chen Mao http://orcid.org/0000-0002-6537-6215

\section{REFERENCES}

1 Safiri S, Mansournia AM. Associations of regular glucosamine use with all-cause and cause-specific mortality: causality assumptions need to be checked. Ann Rheum Dis 2020. 10.1136/annrheumdis-2020-217732

2 Li Z-H, Gao X, Chung VC, et al. Associations of regular glucosamine use with all-cause and cause-specific mortality: a large prospective cohort study. Ann Rheum Dis 2020. doi:10.1136/annrheumdis-2020-217176. [Epub ahead of print: 06 Apr 2020].

3 Kantor ED, Lampe JW, Navarro SL, et al. Associations between glucosamine and chondroitin supplement use and biomarkers of systemic inflammation. J Altern Complement Med 2014;20:479-85.

$4 \mathrm{Ma} \mathrm{H}, \mathrm{LiX}$, Sun D, et al. Association of habitual glucosamine use with risk of cardiovascular disease: prospective study in UK Biobank. BMJ 2019;365:11628.

5 Bell GA, Kantor ED, Lampe JW, et al. Use of glucosamine and chondroitin in relation to mortality. Eur J Epidemiol 2012;27:593-603. 
Table 1 Baseline characteristics of study participants by glucosamine use

\begin{tabular}{|c|c|c|c|c|}
\hline Characteristics & $\begin{array}{l}\text { Overall } \\
(\mathrm{N}=495077)\end{array}$ & $\begin{array}{l}\text { Glucosamine non-users } \\
(n=400731)\end{array}$ & $\begin{array}{l}\text { Glucosamine users } \\
(n=94346)\end{array}$ & SMD \\
\hline Age, mean (SD), years & $56.55(8.09)$ & $55.95(8.20)$ & $59.08(7.07)$ & 0.409 \\
\hline Women & $269549(54.4)$ & $210497(52.5)$ & 59052 (62.6) & 0.205 \\
\hline TDI, mean (SD) & $-1.31(3.09)$ & $-1.20(3.14)$ & $-1.79(2.79)$ & 0.201 \\
\hline Education & & & & 0.017 \\
\hline Degree & $160288(32.4)$ & $129146(32.2)$ & $31142(33.0)$ & \\
\hline No degree & $334789(67.6)$ & $271585(67.8)$ & $63204(67.0)$ & \\
\hline Ethnicity & & & & 0.085 \\
\hline White & $455861(92.1)$ & $367313(91.7)$ & 88548 (93.9) & \\
\hline Others & $39216(7.9)$ & $33418(8.3)$ & $5798(6.1)$ & \\
\hline \multicolumn{5}{|l|}{ Household income (f) } \\
\hline$<18000$ & $116815(23.6)$ & $95680(23.9)$ & $21135(22.4)$ & 0.094 \\
\hline $18000-30999$ & $127517(25.8)$ & $100419(25.1)$ & $27098(28.7)$ & \\
\hline $31000-51999$ & $127427(25.7)$ & $102879(25.7)$ & $24548(26.0)$ & \\
\hline 52 000-100 000 & 97565 (19.7) & $80314(20.0)$ & $17251(18.3)$ & \\
\hline$>100000$ & $25753(5.2)$ & $21439(5.3)$ & $4314(4.6)$ & \\
\hline BMI, mean (SD), $\mathrm{kg} / \mathrm{m}^{2}$ & $27.43(4.80)$ & $27.45(4.84)$ & $27.36(4.65)$ & 0.02 \\
\hline Smoking status & & & & 0.184 \\
\hline Never & $271144(54.8)$ & $219107(54.7)$ & $52037(55.2)$ & \\
\hline Former & $171668(34.7)$ & $135486(33.8)$ & $36182(38.4)$ & \\
\hline Current & $52265(10.6)$ & $46138(11.5)$ & $6127(6.5)$ & \\
\hline Alcohol consumption & & & & 0.081 \\
\hline Never & $21931(4.4)$ & $18688(4.7)$ & $3243(3.4)$ & \\
\hline Former & $17858(3.6)$ & $15136(3.8)$ & $2722(2.9)$ & \\
\hline Current & $455288(92.0)$ & $366907(91.6)$ & $88381(93.7)$ & \\
\hline Physical activity (min/week) & & & & 0.137 \\
\hline$<150$ & $228019(46.1)$ & $189753(47.4)$ & $38266(40.6)$ & \\
\hline$\geq 150$ & $267058(53.9)$ & $210978(52.6)$ & $56080(59.4)$ & \\
\hline Vegetable consumption (servings/day) & & & & 0.167 \\
\hline$<2.0$ & $97853(19.8)$ & $83776(20.9)$ & $14077(14.9)$ & \\
\hline $2.0-3.9$ & $222743(45.0)$ & $179783(44.9)$ & $42960(45.5)$ & \\
\hline$\geq 4.0$ & $174481(35.2)$ & $137172(34.2)$ & 37309 (39.5) & \\
\hline Fruit consumption (servings/day) & & & & 0.286 \\
\hline$<2.0$ & $136458(27.6)$ & $118612(29.6)$ & $17846(18.9)$ & \\
\hline $2.0-3.9$ & $201446(40.7)$ & $163136(40.7)$ & $38310(40.6)$ & \\
\hline$\geq 4.0$ & $157173(31.7)$ & $118983(29.7)$ & $38190(40.5)$ & \\
\hline \multicolumn{5}{|l|}{ Supplement or drug use } \\
\hline Vitamin & $157133(31.7)$ & $104719(26.1)$ & $52414(55.6)$ & 0.627 \\
\hline Minerals and other dietary supplements & $184377(37.2)$ & $118971(29.7)$ & $65406(69.3)$ & 0.864 \\
\hline Aspirin & $66052(13.3)$ & $53402(13.3)$ & $12650(13.4)$ & 0.002 \\
\hline Statin & $56544(11.4)$ & $46186(11.5)$ & $10358(11.0)$ & 0.017 \\
\hline Non-aspirin NSAIDs & $71109(14.4)$ & $53152(13.3)$ & $17957(19.0)$ & 0.157 \\
\hline Chondroitin & $7813(1.6)$ & $1581(0.4)$ & $6232(6.6)$ & 0.343 \\
\hline \multicolumn{5}{|l|}{ Health conditions } \\
\hline Cardiovascular disease & $28709(5.8)$ & $24621(6.1)$ & $4088(4.3)$ & 0.081 \\
\hline Cancer & $39659(8.0)$ & $31506(7.9)$ & $8153(8.6)$ & 0.028 \\
\hline Diabetes & $25968(5.2)$ & $22517(5.6)$ & $3451(3.7)$ & 0.093 \\
\hline Hypertension & $279956(56.5)$ & $225247(56.2)$ & $54709(58.0)$ & 0.036 \\
\hline Respiratory diseases & $1957(0.4)$ & $1555(0.4)$ & $402(0.4)$ & 0.006 \\
\hline Digestive diseases & $1417(0.3)$ & $1259(0.3)$ & $158(0.2)$ & 0.030 \\
\hline High cholesterol & 86406 (17.5) & 70408 (17.6) & $15998(17.0)$ & 0.016 \\
\hline Arthritis & $23217(4.7)$ & $15440(3.9)$ & $7777(8.2)$ & 0.185 \\
\hline Dementia & $219(0.0)$ & $184(0.0)$ & $35(0.0)$ & 0.004 \\
\hline Depression & $76642(15.5)$ & 53848 (13.4) & $15133(16.0)$ & 0.073 \\
\hline Long-standing illness & $162123(32.7)$ & $131024(32.7)$ & $31099(33.0)$ & 0.006 \\
\hline
\end{tabular}

Values are numbers (\%) unless stated otherwise.

BMI, body mass index; NSAID, non-steroidal anti-inflammatory drug; SMD, standardised mean difference; TDI, Townsend Deprivation Index. 